\title{
Review
}

\section{Studies on stress distribution in pavements subjected to surface shear forces}

\author{
By Tsutomu KImURA ${ }^{* 1, * 2, \dagger}$
}

(Communicated by Kiyoshi HoRIKAwA, M.J.A.)

\begin{abstract}
It has been pointed out by some researchers ${ }^{1), 2)}$ that road pavements are subjected to vertical stress due to vehicles on them as well as shear stress at the time of braking or acceleration of vehicles. In this paper, the results of elastic analysis to obtain the rigorous solution for an elastic two-layer system subjected to surface shear stress are described and it is shown that the effect of shear stresses applied at the surface gives rise to fairly large stresses in the system. On the basis of these findings, the author attempts to explain why pavement failure takes place frequently at places such as crossings and curved parts where pavements are subjected to high magnitude of surface shear stresses.
\end{abstract}

Keywords: three-dimensional elasticity analysis, two-layer system, surface shear force, crossings and curved parts of road pavements, pavement failure

\section{Fundamental solutions}

In a three-dimensional elastic half-space, elastic equilibrium equations in terms of displacement components $u_{r}, u_{\theta}, u_{z}$ in an axi-symmetrical coordinate $r, \theta, z$ can be written as follows. They are

$$
\left.\begin{array}{l}
\nabla_{p^{2}} u_{r}+\frac{1}{1-2 \nu} \frac{\partial e_{p}}{\partial r}-\frac{1}{r}\left\{\frac{2}{r} \frac{\partial u_{\theta}}{\partial \theta}+\frac{u_{r}}{r}\right\}=0 \\
\nabla_{p^{2}} u_{\theta}+\frac{1}{1-2 \nu r} \frac{\partial e_{p}}{\partial \theta}-\frac{1}{r}\left\{\frac{u_{\theta}}{r}-\frac{2}{r} \frac{\partial u_{r}}{\partial \theta}\right\}=0 \\
\nabla_{p^{2}} u_{z}+\frac{1}{1-2 \nu} \frac{\partial e_{p}}{\partial z}=0
\end{array}\right\}
$$

where $\nabla_{p^{2}}=\left(\frac{\partial^{2}}{\partial r^{2}}+\frac{1}{r} \frac{\partial}{\partial r}+\frac{1}{r^{2}} \frac{\partial^{2}}{\partial \theta^{2}}+\frac{\partial^{2}}{\partial z^{2}}\right)$ and $e_{p}=\frac{\partial u_{r}}{\partial r}+$ $\frac{\partial u_{\theta}}{r \partial \theta}+\frac{u_{r}}{r}+\frac{\partial u_{z}}{\partial z}$.

Expressing the displacement components $u_{r}, u_{\theta}$, $u_{z}$ by two displacement functions $\Phi$ and $\Psi$, and denoting Poission's ratio as $\nu$, one obtains ${ }^{3)}$

$$
\left.\begin{array}{l}
u_{r}=-\frac{\partial^{2} \Phi}{\partial r \partial z}+\frac{2}{r} \frac{\partial \Psi}{\partial \theta} \\
u_{\theta}=-\frac{\partial^{2} \Phi}{r \partial \theta \partial z}-2 \frac{\partial \Psi}{\partial r} \\
u_{z}=2(1-\nu) \nabla_{p^{2}} \Phi-\frac{\partial^{2} \Phi}{\partial z^{2}}
\end{array}\right\}
$$

*1 Professor Emeritus, Tokyo Institute of Technology.

*2 Recipient of the Japan Academy Prize in 2012.

$\dagger \quad$ Correspondence should be addressed: T. Kimura, 3-14-6 Minami-no, Hachiohji, Tokyo 192-0916, Japan (e-mail: tomkim@ muj.biglobe.ne.jp).
Substituting these into Eq. [1-1], the following two fundamental equations are obtained. They are

$$
\nabla_{p^{4}} \Phi=0, \quad \nabla_{p^{2}} \Psi=0
$$

where $\nabla_{p^{4}}=\left(\frac{\partial^{2}}{\partial r^{2}}+\frac{1}{r} \frac{\partial}{\partial r}+\frac{1}{r^{2}} \frac{\partial^{2}}{\partial \theta^{2}}+\frac{\partial^{2}}{\partial z^{2}}\right)^{2}$.

Using these two displacement functions, six stress components in the elastic half-space are written as ${ }^{3)}$

$$
\left.\begin{array}{rl}
\frac{\sigma_{r}}{2 \mu}= & \frac{\partial}{\partial z}\left(\nu \nabla_{p^{2}} \Phi-\frac{\partial^{2} \Phi}{\partial r^{2}}\right)+\frac{2}{r} \frac{\partial^{2} \Psi}{\partial \theta \partial r}-\frac{2}{r^{2}} \frac{\partial \Psi}{\partial \theta} \\
\frac{\sigma_{\theta}}{2 \mu}= & \frac{\partial}{\partial z}\left(\nu \nabla_{p^{2}} \Phi-\frac{1}{r} \frac{\partial \Phi}{\partial r}-\frac{1}{r^{2}} \frac{\partial^{2} \Phi}{\partial \theta^{2}}\right) \\
& -\frac{2}{r} \frac{\partial^{2} \Psi}{\partial \theta \partial r}+\frac{2}{r^{2}} \frac{\partial \Psi}{\partial \theta} \\
\frac{\sigma_{z}}{2 \mu}= & \frac{\partial}{\partial z}\left\{(2-\nu) \nabla_{p^{2}} \Phi-\frac{\partial^{2} \Phi}{\partial z^{2}}\right\} \\
\frac{\tau_{\theta z}}{2 \mu}= & \frac{\partial}{r \partial \theta}\left\{(1-\nu) \nabla_{p^{2}} \Phi-\frac{\partial^{2} \Phi}{\partial z^{2}}\right\}-\frac{\partial^{2} \Psi}{\partial r \partial z} \\
\frac{\tau_{z r}}{2 \mu}= & \frac{\partial}{\partial r}\left\{(1-\nu) \nabla_{p^{2}} \Phi-\frac{\partial^{2} \Phi}{\partial z^{2}}\right\}+\frac{\partial^{2} \Psi}{r \partial \theta \partial z} \\
\frac{\tau_{\theta r}}{2 \mu}= & \frac{\partial^{2}}{r \partial \theta \partial z}\left\{\frac{\Phi}{r}-\frac{\partial \Phi}{\partial r}\right\}-2 \frac{\partial^{2} \Psi}{\partial r^{2}}-\frac{\partial^{2} \Psi}{\partial z^{2}}
\end{array}\right\}
$$

where $\mu$ is the Lame's constant and $\nu$ is the Poisson's ratio.

The technique of separation of variables enables us to express the two displacement functions as 


$$
\left.\begin{array}{l}
\Phi=\sum_{m=0}^{\infty} \Phi_{m}(r, z) \cos m \theta \\
\Psi=\sum_{m=0}^{\infty} \Psi_{m}(r, z) \sin m \theta
\end{array}\right\}
$$

Denoting the Hankel transforms of $\Phi_{m}$ and $\Psi_{m}$ as ${ }^{4)}$

$$
\left.\begin{array}{l}
G_{m}(\xi, z)=\int_{0}^{\infty} r \Phi_{m}(r, z) J_{m}(\xi r) d r \\
H_{m}(\xi, z)=\int_{0}^{\infty} r \Psi_{m}(r, z) J_{m}(\xi r) d r
\end{array}\right\}
$$

where $J_{m}(\xi r)$ is the Bessel function of first kind.

Putting these into Eq. [1-3] leads to a set of ordinary differential equations

$$
\left.\begin{array}{l}
\left(\frac{d^{2}}{d z^{2}}-\xi^{2}\right)^{2} G_{m}(\xi, z)=0 \\
\left(\frac{d^{2}}{d z^{2}}-\xi^{2}\right) H_{m}(\xi, z)=0
\end{array}\right\}
$$

for which solutions are

$$
\left.\begin{array}{l}
G_{m}(\xi, z)=\left(A_{m}+B_{m} z\right) e^{\xi z}+\left(C_{m}+D_{m} z\right) e^{-\xi z} \\
H_{m}(\xi, z)=E_{m} e^{\xi z}+F_{m} e^{-\xi z}
\end{array}\right\}
$$

where $A_{m}, B_{m},,, F_{m}$ are constants to be determined from boundary conditions.

Inverse Hankel transforms of Eq. [1-6] gives $\Phi_{m}(r, z)$ and $\Psi_{m}(r, z)$ as

$$
\left.\begin{array}{l}
\Phi_{m}(r, z)=\int_{0}^{\infty} \xi G_{m}(\xi, z) J_{m}(\xi r) d \xi \\
\Psi_{m}(r, z)=\int_{0}^{\infty} \xi H_{m}(\xi, z) J_{m}(\xi r) d \xi
\end{array}\right\}
$$

Substituting these into Eqs. [1-2] and [1-4], all the displacement and stress components can be derived as

$$
\begin{aligned}
u_{r}= & \frac{1}{2} \sum_{m=0}^{\infty}\left[\int _ { 0 } ^ { \infty } \left\{\left(A_{m} \xi+B_{m} z \xi+B_{m}+2 E_{m}\right) e^{\xi z}\right.\right. \\
& \left.-\left(C_{m} \xi+D_{m} z \xi-D_{m}-2 F_{m}\right) e^{-\xi z}\right\} \xi^{2} J_{m+1}(\xi r) d \xi \\
& -\int_{0}^{\infty}\left\{\left(A_{m} \xi+B_{m} z \xi+B_{m}-2 E_{m}\right) e^{\xi z}\right. \\
& \left.\left.-\left(C_{m} \xi+D_{m} z \xi-D_{m}+2 F_{m}\right) e^{-\xi z}\right\} \xi^{2} J_{m-1}(\xi r) d \xi\right] \cos m \theta \\
u_{\theta}= & \frac{1}{2} \sum_{m=0}^{\infty}\left[\int _ { 0 } ^ { \infty } \left\{\left(A_{m} \xi+B_{m} z \xi+B_{m}+2 E_{m}\right) e^{\xi z}\right.\right. \\
& \left.-\left(C_{m} \xi+D_{m} z \xi-D_{m}-2 F_{m}\right) e^{-\xi z}\right\} \xi^{2} J_{m+1}(\xi r) d \xi \\
& +\int_{0}^{\infty}\left\{\left(A_{m} \xi+B_{m} z \xi+B_{m}-2 E_{m}\right) e^{\xi z}\right. \\
& \left.\left.-\left(C_{m} \xi+D_{m} z \xi-D_{m}+2 F_{m}\right) e^{-\xi z}\right\} \xi^{2} J_{m-1}(\xi r) d \xi\right] \sin m \theta \\
u_{z}= & \frac{1}{2} \sum_{m=0}^{\infty}\left[\int _ { 0 } ^ { \infty } \left\{\left(-A_{m} \xi^{2}+2 B_{m} \xi \overline{1-2 \nu}-B_{m} z \xi^{2}\right) e^{\xi z}\right.\right. \\
& \left.\left.-\left(C_{m} \xi^{2}+2 D_{m} \xi \overline{1-2 \nu}+D_{m} z \xi^{2}\right) e^{-\xi z}\right\} \xi J_{m}(\xi r) d \xi\right] \cos m \theta
\end{aligned}
$$

$$
\begin{aligned}
& \sigma_{r}=2 \mu \sum_{m=0}^{\infty}\left[\int _ { 0 } ^ { \infty } \left\{\left(A_{m} \xi^{3}+B_{m} \xi^{2} \overline{1+2 \nu}+B_{m} z \xi^{3}\right) e^{\xi z}\right.\right. \\
& \left.-\left(C_{m} \xi^{2}-D_{m} \xi^{2} \overline{1+2 \nu}+D_{m} z \xi^{3}\right) e^{-\xi z}\right\} \xi J_{m}(\xi r) d \xi \\
& -\frac{m+1}{2 r} \int_{0}^{\infty}\left\{\left(A_{m} \xi+B_{m} z \xi+B_{m}+2 E_{m}\right) e^{\xi z}\right. \\
& \left.-\left(C_{m} \xi+D_{m} z \xi-D_{m}+2 F_{m}\right) e^{-\xi z}\right\} \xi^{2} J_{m+1}(\xi r) d \xi \\
& -\frac{m-1}{2 r} \int_{0}^{\infty}\left\{\left(A_{m} \xi+B_{m} z \xi+B_{m}+2 E_{m}\right) e^{\xi z}\right. \\
& \left.\left.-\left(C_{m} \xi+D_{m} z \xi-D_{m}+2 F_{m}\right) e^{-\xi z}\right\} \xi^{2} J_{m-1}(\xi r) d \xi\right] \cos m \theta \\
& \sigma_{\theta}=2 \mu \sum_{m=0}^{\infty}\left[\nu \int_{0}^{\infty}\left(2 B_{m} \xi^{2} e^{\xi z}+2 D_{m} \xi^{2} e^{-\xi z}\right) \xi J_{m}(\xi z) d \xi\right. \\
& +\frac{m+1}{2 r} \int_{0}^{\infty}\left\{\left(A_{m} \xi+B_{m} z \xi+B_{m}+2 E_{m}\right) e^{\xi z}\right. \\
& \left.-\left(C_{m} \xi+D_{m} z \xi-D_{m}-2 F_{m}\right) e^{-\xi z}\right\} \xi^{2} J_{m+1}(\xi r) d \xi \\
& +\frac{m-1}{2 r} \int_{0}^{\infty}\left\{\left(A_{m} \xi+B_{m} z \xi+B_{m}-2 E_{m}\right) e^{\xi z}\right. \\
& \left.\left.-\left(C_{m} \xi+D_{m} z \xi-D_{m}+2 F_{m}\right) e^{-\xi z}\right\} \xi^{2} J_{m-1}(\xi r) d \xi\right] \cos m \theta \\
& \sigma_{z}=2 \mu \sum_{m=0}^{\infty}\left[\int _ { 0 } ^ { \infty } \left\{\left(-A_{m} \xi+B_{m} \overline{1-2 \nu}-B_{m} z \xi\right) e^{\xi z}\right.\right. \\
& \left.\left.+\left(C_{m} \xi+D_{m} \overline{1-2 \nu}+D_{m} z \xi\right) e^{-\xi z}\right\} \xi^{3} J_{m}(\xi r) d \xi\right] \cos m \theta \\
& \left.\begin{array}{l}
\tau_{\theta z} \\
\tau_{r z}
\end{array}\right\}=\mu \sum_{m=0}^{\infty}\left[\int _ { 0 } ^ { \infty } \left\{\left(A_{m} \xi+B_{m} \overline{2 \nu+\xi z}+E_{m}\right) e^{\xi z}\right.\right. \\
& \left.+\left(C_{m} \xi-D_{m} \overline{2 \nu-\xi z}-F_{m}\right) e^{-\xi z}\right\} \xi^{3} J_{m+1}(\xi r) d \xi \\
& \pm \int_{0}^{\infty}\left\{\left(A_{m} \xi+B_{m} \overline{2 \nu+\xi z}-E_{m}\right) e^{\xi z}\right. \\
& \left.\left.+\left(C_{m}-D_{m} \overline{2 \nu-\xi z}+F_{m}\right) e^{-\xi z}\right\} J_{m-1}(\xi r) d \xi\right]\left(\begin{array}{c}
\sin m \theta \\
\cos m \theta
\end{array}\right) \\
& \tau_{r \theta}=\mu \sum_{m=0}^{\infty}\left[\int_{0}^{\infty}\left(E_{m} e^{\xi z}+F_{m} e^{-\xi z}\right) \xi^{3} J_{m}(\xi r) d \xi\right. \\
& -\frac{m+1}{2 r} \int_{0}^{\infty}\left\{\left(A_{m} \xi+B_{m} z \xi+B_{m}+2 E_{m}\right) e^{\xi z}\right. \\
& \left.-\left(C_{m} \xi+D_{m} z \xi-D_{m}-2 F_{m}\right) e^{-\xi z}\right\} \xi^{2} J_{m+1}(\xi r) d \xi \\
& +\frac{m-1}{2 r} \int_{0}^{\infty}\left\{\left(A_{m} \xi+B_{m} z \xi+B_{m}-2 E_{m}\right) e^{\xi z}\right. \\
& \left.\left.-\left(C_{m} \xi+D_{m} z \xi-D_{m}+2 F_{m}\right) e^{-\xi z}\right\} \xi^{2} J_{m-1}(\xi r) d \xi\right] \sin m \theta
\end{aligned}
$$

\section{Stress analyses for a two layer system subjected to surface shear force}

The boundary conditions when shear stress is applied to the surface of pavement by a vehicle as shown in Fig. 1 can be written as follows. ${ }^{4)}$ They are

at $z=-h$

$$
\left.\begin{array}{l}
\sigma_{z}=0 \\
\tau_{\theta z}=-P_{0}\left[1-S_{a}(r)\right] \sin \theta \\
\tau_{r z}=P_{0}\left[1-S_{a}(r)\right] \cos \theta
\end{array}\right\}
$$


where $P_{0}=\frac{P}{\pi a^{2}}, \quad S_{a}(r)=\left\{\begin{array}{l}1 ; r<a \\ 0 ; r>a\end{array}\right.$

at $z=0$

$$
\left.\begin{array}{c}
u_{r_{1}}=u_{r_{2}}, \quad u_{\theta_{1}}=u_{\theta_{2}}, \quad u_{z_{1}}=u_{z_{2}} \\
\sigma_{z_{1}}=\sigma_{z_{2}}, \quad \tau_{\theta z_{1}}=\tau_{\theta z_{2}}, \quad \tau_{r z_{1}}=\tau_{r z_{2}}
\end{array}\right\}
$$

Considering the conditions that all the displacement and stress components tend to be zero at infinity, two functions $G_{m}(\xi, z)$ and $H_{m}(\xi, z)$ in Eq. [1-8] are written as
oUpper layer

$$
\left.\begin{array}{l}
G_{m_{1}}(\xi, z)=\left(A_{m_{1}}+B_{m_{1}} z\right) e^{\xi z}+\left(C_{m_{1}}+D_{m_{1}} z\right) e^{-\xi z} \\
H_{m_{1}}(\xi, z)=E_{m_{1}} e^{\xi z}+F_{m_{1}} e^{-\xi z}
\end{array}\right\}
$$

oLower layer

$$
\left.\begin{array}{l}
G_{m_{2}}(\xi, z)=\left(C_{m_{2}}+D_{m_{2}} z\right) e^{-\xi z} \\
H_{m_{2}}(\xi, z)=F_{m_{2}} e^{-\xi z}
\end{array}\right\}
$$

where suffix 1 and 2 refers to the upper and lower layer, respectively.

Nine constants in Eqs. [2-3] and [2-4] can be determined from nine boundary conditions in Eqs. [2-1] and [2-2]. Putting those constants into Eq. [1-10] and assuming $\nu_{1}=\nu_{2}=1 / 2$, stress components $\sigma_{z}, \tau_{\theta z}, \tau_{r z}$ are given as

$$
\begin{aligned}
& \text { ○Upper layer } \\
& \begin{aligned}
\sigma_{z_{1}}= & p P_{0}\left[\int_{0}^{\infty} \frac{N^{2} e^{-2 x+2 x \alpha}(1+\alpha) x-N\left\{\left(x+x \alpha-2 x^{2} \alpha\right) e^{2 x \alpha}+\left(x+x \alpha-2 x^{2} \alpha\right) e^{-2 x}\right\}+x(1+\alpha)}{N^{2} e^{-4 x}-2 N e^{-2 x}\left(2 x^{2}+1\right)+1}\right. \\
& \left.\times e^{-x(1+\alpha)} J_{1}(s x) J_{1}(p x) d x\right] \cos \theta \\
\left.\tau_{\theta_{z_{1}}}\right\}= & \frac{p P_{0}}{2}\left[-\int_{0}^{\infty}\left[\frac{N^{2} e^{-2 x+2 x \alpha}(1+x+x \alpha)+N\left\{\left(2 x^{2} \alpha+x-x \alpha-1\right) e^{2 x \alpha}+\left(2 x^{2} \alpha+x \alpha-x-1\right) e^{-2 x}\right\}+\{1-x(1+\alpha)\}}{N^{2} e^{-4 x}-2 N e^{-2 x}\left(2 x^{2}+1\right)+1}\right.\right. \\
\tau_{r z_{1}} & -\frac{\left.1-N e^{2 \alpha x}\right] e^{-x(1+\alpha)} J_{2}(s x) J_{1}(p x) d x}{\left.1-N e^{-2 x}\right]} \\
& \mp \int_{0}^{\infty}\left[\frac{N^{2} e^{-2 x+2 x \alpha}(1+x+x \alpha)+N\left\{\left(2 x^{2} \alpha+x-x \alpha-1\right) e^{2 x \alpha}+\left(2 x^{2} \alpha+x \alpha-x-1\right) e^{-2 x}\right\}+\{1-x(1+\alpha)\}}{N^{2} e^{-4 x}-2 N e^{-2 x}\left(2 x^{2}+1\right)+1}\right. \\
& \left.\left.+\frac{1-N e^{2 \alpha x}}{1-N e^{-2 x}}\right] e^{-x(1+\alpha) x} J_{0}(s x) J_{1}(p x) d x\right]\left(\begin{array}{c}
\sin \theta \\
\cos \theta
\end{array}\right)
\end{aligned}
\end{aligned}
$$

oLower layer

$$
\begin{aligned}
& \sigma_{z_{2}}=p P_{0}\left[-\int_{0}^{\infty} \frac{N e^{-2 x}\left\{x+x \alpha+2 x^{2} \alpha\right\}-x(1+\alpha)}{N^{2} e^{-4 x}-2 N e^{-2 x}\left(2 x^{2}+1\right)+1}(1-N) e^{-x(1+\alpha)} J_{1}(s x) J_{1}(p x) d x\right] \cos \theta \\
& \left.\begin{array}{c}
\tau_{\theta_{z_{2}}} \\
\tau_{r z_{2}}
\end{array}\right\}=\frac{p P_{0}}{2}\left[-\int_{0}^{\infty}\left[\frac{N e^{-2 x}\left(2 x^{2} \alpha+x \alpha-x-1\right)-(x \alpha+x-1)}{N^{2} e^{-4 x}-2 N e^{-2 x}\left(2 x^{2}+1\right)+1}-\frac{1}{1-N e^{-2 x}}\right]\right. \\
& \times(1-N) e^{-x(1+\alpha)} J_{2}(s x) J_{1}(p x) d x \\
& \mp \int_{0}^{\infty}\left[\frac{N e^{-2 x}\left(2 x^{2} \alpha+x \alpha-x-1\right)-(x \alpha+x-1)}{N^{2} e^{-4 x}-2 N e^{-2 x}\left(2 x^{2}+1\right)+1}+\frac{1}{1-N e^{-2 x}}\right] \\
& \left.\times(1-N) e^{-x(1+\alpha)} J_{0}(s x) J_{1}(p x) d x\right]\left(\begin{array}{c}
\sin \theta \\
\cos \theta
\end{array}\right)
\end{aligned}
$$

where, $N=\frac{E_{1}-E_{2}}{E_{1}+E_{2}}, x=\xi h, \alpha=\frac{z}{h}, s=\frac{r}{h}, p=\frac{a}{h}$, and $E_{1}$ and $E_{2}$ denote the Young's modulus of upper and lower layer.

It has been said that the maximum shearing stress $\tau_{\max }$ in a pavement is one of the factors governing the failure of the pavement. The highest magnitude of $\tau_{\max }$ in pavements subjected to surface shear force appears along the axis of symmetry in Fig. 1, which is equal to $\tau_{r z}$ at $\theta=0$. The maximum $\tau_{\max }$ for vertical surface force can be given by $\left(\sigma_{z}-\sigma_{r}\right) / 2 .^{5)}$ The maximum shearing stresses along the axis of symmetry are follows. 
a) Surface shear force

-Upper layer

$$
\begin{aligned}
\tau_{\max }= & \frac{p P_{0}}{2} \int_{0}^{\infty}\left[\frac{N^{2} e^{-2 x+2 x \alpha}(1+x+x \alpha)+N\left\{\left(2 x^{2} \alpha+x-x \alpha-1\right) e^{2 x \alpha}+\left(2 x^{2} \alpha-x+x \alpha-1\right) e^{-2 x}\right\}+\{1-x(1+\alpha)\}}{N^{2} e^{-4 x}-2 N e^{-2 x}\left(2 x^{2}+1\right)+1}\right. \\
& \left.+\frac{1-N e^{2 \alpha x}}{1-N e^{-2 x}}\right] \times e^{-x(1+\alpha)} J_{1}(p x) d x
\end{aligned}
$$

-Lower layer

$$
\left.\tau_{\max }=\frac{p P_{0}}{2} \int_{0}^{\infty}\left[\frac{N e^{-2 x}\left(2 x^{2} \alpha+x \alpha-x-1\right)-(x \alpha+x-1)}{N^{2} e^{-4 x}-2 N e^{-2 x}\left(2 x^{2}+1\right)+1}+\frac{1}{1-N e^{-2 x}}\right] \times(1-N) e^{-x(1+\alpha)} J_{1}(p x) d x\right]
$$

b) Surface vertical force

$$
\begin{aligned}
& \text { ○Upper layer } \\
& \begin{aligned}
\tau_{\max }= & \frac{3 p P_{0}}{4} \int_{0}^{\infty}\left[\frac{N^{2} e^{-2 x+2 x \alpha}(x+x \alpha)-N\left\{\left(2 x^{2} \alpha+x+x \alpha\right) e^{2 x \alpha}+\left(2 x^{2} \alpha-x-x \alpha\right) e^{-2 x}\right\}-x(1+\alpha)}{N^{2} e^{-4 x}-2 N e^{-2 x}\left(2 x^{2}+1\right)+1}\right] \\
& \times e^{-x(1+\alpha)} J_{1}(p x) d x
\end{aligned}
\end{aligned}
$$

oLower layer

$\tau_{\max }=\frac{3 p P_{0}}{4} \int_{0}^{\infty}\left[\frac{N e^{-2 x}\left(2 x^{2} \alpha-x-x \alpha\right)+x(1+\alpha)}{N^{2} e^{-4 x}-2 N e^{-2 x}\left(2 x^{2}+1\right)+1}\right](1-N) e^{-x(1+\alpha)} J_{1}(p x) d x$

\section{Numerical analysis and discussions on the results}

Numerical analysis. In order to visualize the shear stress distributions in two layer systems subjected to surface shear force, numerical integrations were carried out on Eqs. [2-5] and [2-6]. The results are shown in Fig. 2 for the cases $p=1,2$ and $E_{1} / E_{2}=2,10,100$. Numerical integrations were also conducted on Eqs. [2-7] and [2-8] in an attempt to investigate the differences of the effect of $\tau_{\max }$ on pavement failure for surface shear forces and vertical forces. The results are illustrated in Fig. 3.

\section{Discussions on the results.}

A) Stress distributions.

1. Unlike the vertical stresses in the two layer systems subjected to vertical force at the surface, shear stresses in the elastic medium subjected to surface shear force do not propagate to deeper areas but tend to spread to the horizontal directions.

2 . One of the shear stress components $\tau_{\theta z}$ does not spread to the horizontal direction as much as $\tau_{r z}$.

3. For the case of two layer systems subjected to vertical force, Burmister, D.M. ${ }^{6)-8}$ found that the stresses tend to concentrate in the upper layer and that the extent of concentration becomes higher for higher ratios of Young's modulus $E_{1} / E_{2}$. The stress

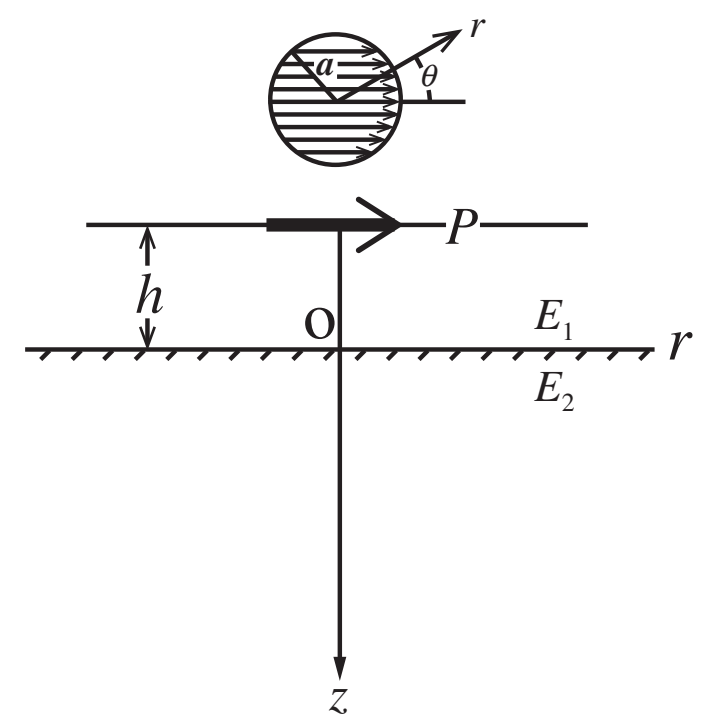

Fig. 1. Axisymmetric polar coordinate used in the analysis; $a$ and $P$ denote the radius of loaded area and total shear force respectively.

concentration takes place also for the case of surface shear force, and the extent is more conspicuous for the same ratio of Young's modulus. It is interesting to notice, however, that the extent of spreading of 


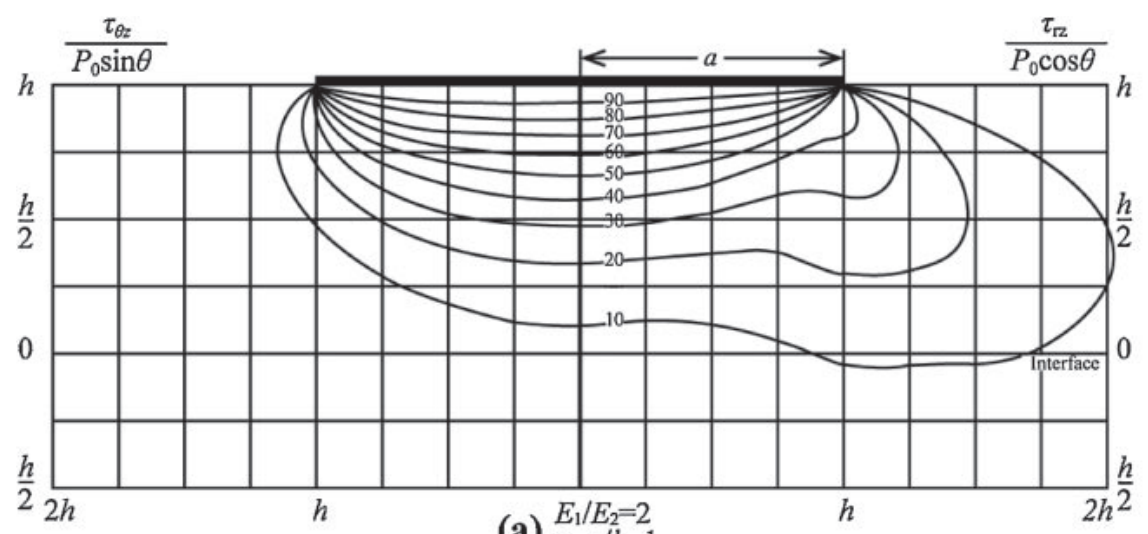

(a) $\begin{gathered}E_{1} / E_{2}=2 \\ p=a / h=1\end{gathered}$
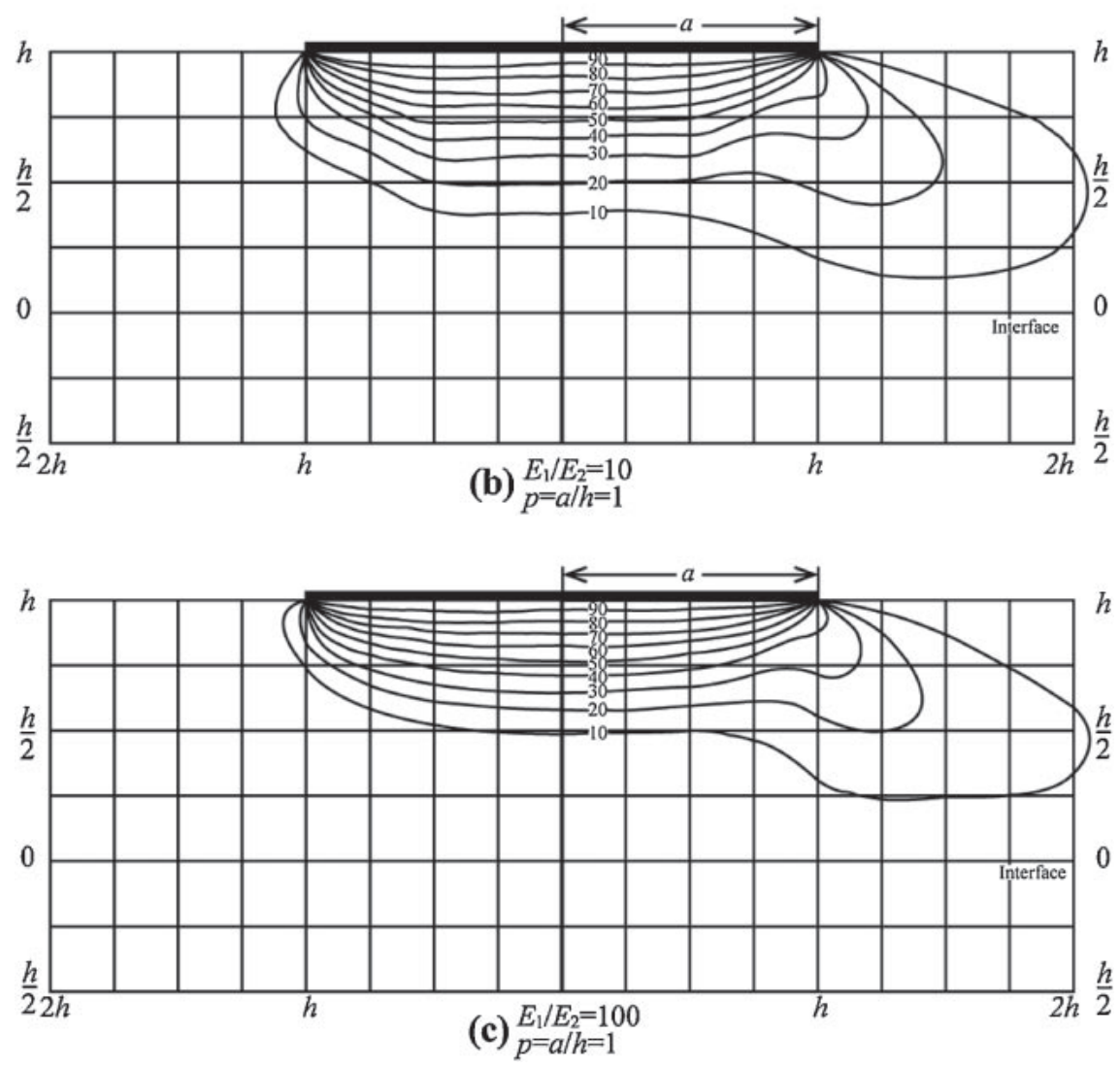

Fig. 2. Continued.

stresses towards horizontal direction does not vary very much with the Young's modulus ratio.

4. For two layer systems with a thinner upper layer, propagation of shear stresses into vertical direction becomes less noticeable, while spreading towards horizontal direction does not show so much difference and the whole stress distribution pattern is not much influenced by the magnitude of stressed area at the surface for higher Young's modulus ratios.
5. Stress contours of shear stress component $\tau_{r z}$ at lower stress levels, tend to go deeper at places some distance away from the axis of symmetry. This implies that at a horizontal plane at considerable depth the shear stress $\tau_{r z}$ assumes the maximum value in these areas. Barber, E.S. ${ }^{1)}$ pointed out that this stress is likely to act as the stress to tear the upper layer from the lower layer of a pavement. He stressed the importance of adding the effect of this 

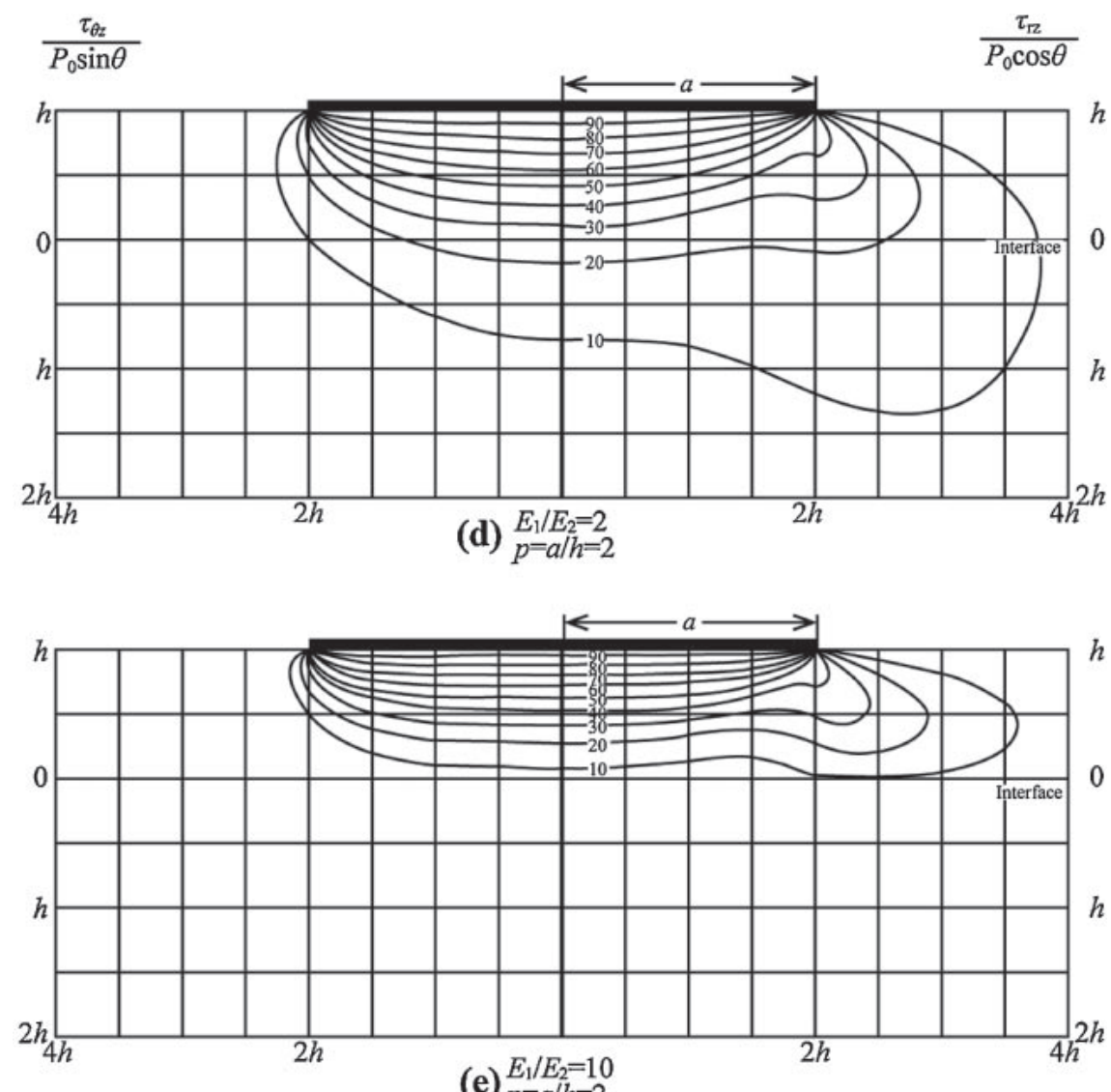

(e) $\begin{aligned} & E_{1} / E_{2}=10 \\ & p=a / h=2\end{aligned}$

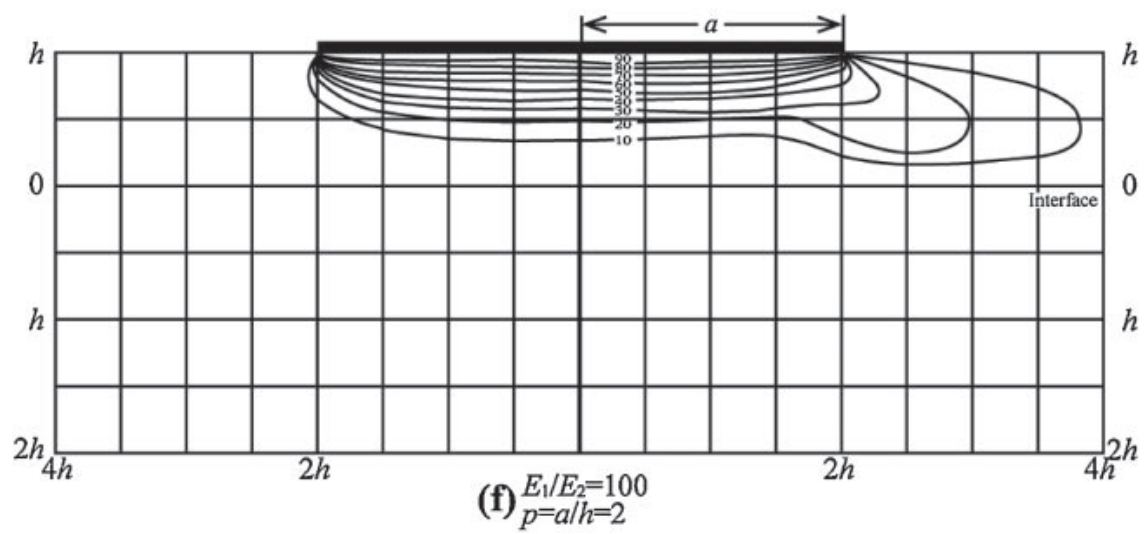

Fig. 2. Pressure bulbs for $\tau_{r z}$ and $\tau_{\theta z}$ for two-layer systems subjected to surface shear force. (Figures on the stress contour lines indicate the percentage of the shear stress on each contour line to the surface shear force.)

stress to the stress caused by vertical vehicle load in designing the bonding stress at the interface of pavements.

B) The maximum shearing stresses in pavements.

1. On the contrary to two layer systems subjected to vertical surface force in which the distributions of the maximum shearing stress vary very much with the Young's modulus ratio $E_{1} / E_{2}$, the maximum shearing stresses $\tau_{\max }$ for the cases of surface shear force tend to concentrate in the upper layer irrespective of the magnitude of $E_{1} / E_{2}$.

2 . For vertical surface force, $\tau_{\max }$ in the upper layer varies very much with the depth of the upper layer irrespective of the values of $E_{1} / E_{2}$ and $\tau_{\max }$ in 


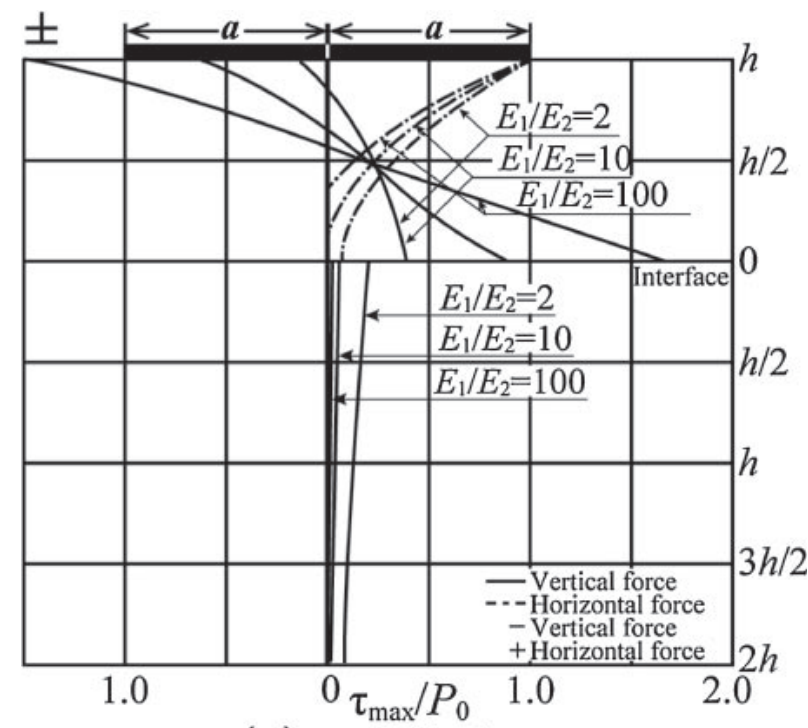

(a) $p=a / h=1$

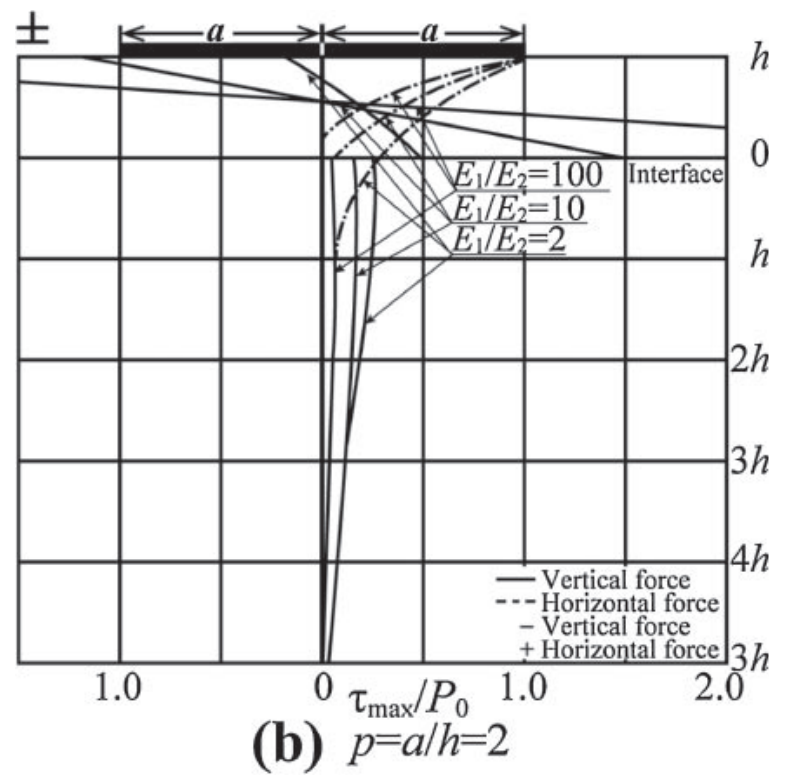

Fig. 3. Maximum shearing stress $\tau_{\max }$ in two-layer systems subjected to surface shear force/surface vertical force.

the lower layer varies with the depth of the upper layer for higher values of $E_{1} / E_{2}$. This is not the case for surface shear force, which may lead to a conclusion that increasing the depth of upper layer is not a very effective measure to protect pavements from the damage to be caused by surface shear force. However, for surface vertical force, $\tau_{\max }$ in the upper layer increases very much for higher values of $E_{1} / E_{2}$, although $\tau_{\max }$ in the lower layer decreases. This implies that the use of a rigid upper layer does not necessarily allow engineers to reduce the thickness of the upper layer.

3. Takeshita ${ }^{9)}$ proposed an empirical method to determine the thickness of asphalt (upper) layer of a pavement based on $\tau_{\max }$ along the axis of symmetry of a semi-infinite elastic medium subjected to surface vertical stress $P_{0}$. He suggested that $\tau_{\max }$ in the subgrade (lower layer) should be less than $80 \%$ of maximum value of $\tau_{\max } 289 P_{0} .{ }^{5)}$ His proposal gives $1 / p=h / a=1.51$ which means the thickness of the upper layer $h$ as 1.51a. Simplifying pavements as a two-layer system, the magnitude of $E_{1} / E_{2}$ is considered to be roughly 2-20 for asphalt pavements, for which cases Fig. 3 shows that $\tau_{\max }$ is $0.15-0.18 P_{0}$. Based on these figures, it can be said that Takeshita's proposal gives a considerable overestimation. Reasonable estimation will be somewhere around $h=a$.

4. The Young's modulus ratio $E_{1} / E_{2}$ for concrete pavements is said to be in the range 100-500, while for asphalt pavements it is roughly 2-20 as was described above. Figure 3(a) and (b) show that $\tau_{\max }$ in the upper layer is higher for concrete pavements subjected to surface vertical force than for asphalt pavements receiving surface shear force. However, $\tau_{\max }$ is higher in the lower layer in the vicinity of the interface for asphalt pavements subjected to surface shear force than concrete pavements receiving surface vertical force. This implies that surface vertical force is responsible for failure of concrete pavements and that surface shear force contributes very much to the failure of asphalt pavements. This explains why the failure of asphalt pavements takes place very often at crossings and curved parts of asphalt pavements where high surface shear force is likely to be applied. The importance of surface shear force for asphalt pavements was not appreciated very much up until some years ago, but the road designers gradually started understanding the importance of the effect by referring to the results of this study and subsequent studies in the same line. This has led to the improvement of the asphalt pavement design manuals and to dramatic reduction in failure of asphalt pavements.

5. One of the convenient measures for repairing damaged asphalt pavements is so-called "overlaying"; covering an existing asphalt layer by a thin layer of asphalt mixture. At the time when the effect of surface shear force was not properly taken into account into the design, frequent failures of the overlaying layer took place. In recent years, however, this is not the case, as engineers have understood the mechanism that the surface shear force produces 
fairly large $\tau_{\max }$ at the interface between the overlaid layer and the existing asphalt layer as was found out by this research.

\section{Concluding remarks}

Conclusions derived from this study are summarized as follows.

1. Shear stresses in elastic two-layer systems subjected to surface shear force do not propagate to deeper areas but tend to spread to the horizontal directions. The extent of horizontal spreading does not vary very much with the magnitude of Young's modulus ratio.

2. One of the shear stress components $\tau_{\theta z}$ does not spread to the horizontal directions as much as $\tau_{r z}$. The shear stress component $\tau_{r z}$ at a horizontal plane assumes the maximum value in the areas below the edge of the surface force in deeper areas.

3. The extent of vertical propagation of shearing stresses in elastic two-layer systems subjected to surface shear force becomes smaller for higher magnitudes of the ratio of radius of loaded area to the thickness of the upper layer, but the extent of horizontal spreading does not change very much with the ratio.

4. There are marked differences between the patterns of distribution of the maximum shear stresses in two-layer systems subjected to surface shear force and in those subjected to surface vertical force. The former tends to concentrate to the upper layer irrespective of the magnitude of Young's modulus ratio, but for the latter, the extent of concentration varies very much with the magnitude of Young's modulus ratio.

5. It has been said that pavements at crossings as well as curved parts are subjected to surface shear force of which magnitude is similar to that of surface vertical force coming from the weight of vehicles. The results of this study shows that the maximum shear stresses brought about by surface shear force in the areas near the surface of the upper layers are higher than those by surface vertical force for lower Young's modulus ratios which is the case for asphalt pavements. This implies that the effect of surface shear force should be carefully considered in designing asphalt pavements.

\section{References}

1) Barber, E.S. (1962) Shear loads on pavements. Proceedings of 1st International Conference on Structural Design of Asphalt Pavements, Michigan.

2) Marwick, A.H.D. and Starks, H.J.H. (1941) Stresses between tire and road. J. ICE 16, 309-325.

3) Sneddon, I.N. (1951) Fourier Transforms. McGrawHill, New York.

4) Muki, R. (1956) A three-dimensional elastic problem in which a semi-infinite elastic medium is subjected to surface shear force. Trans. Jpn. Soc. Mech. Eng. 22, 468-474 (in Japanese).

5) Kimura, T. (1978) Stress Distributions in Soil Media. Kajima Publishing Co., Tokyo (in Japanese).

6) Burmister, D.M. (1945) The general theory of stresses and displacements in layered systems. I. J. Appl. Phys. 16, 89-94.

7) Burmister, D.M. (1945) The general theory of stresses and displacements in layered soil systems. II. J. Appl. Phys. 16, 126-127.

8) Burmister, D.M. (1945) The general theory of stresses and displacements in layered soil systems. III. J. Appl. Phys. 16, 296-302.

9) Takeshita, H. (1965) Utilization of a logarithmic graph for the design of asphalt pavement. Road Eng. Man. Rev. No. 295, 726-731 (in Japanese).

(Received July 11, 2013; accepted Dec. 19, 2013) 


\section{Profile}

Tsutomu Kimura was born in 1938. He graduated from Civil Engineering Department, Faculty of Engineering at The University of Tokyo in 1961. After he had worked at a contractor, Nippo Corporation, for one year, he was awarded a study leave to acquire Master degree at The University of Tokyo. Having seen many cases of pavement failure in field, he came to have interests in pavement analyses. Until that time, nobody had been able to explain quantitatively why pavements fail very frequently at the places such as crossing and curved parts where the pavements are subjected to high surface shear in addition to vertical force. He challenged this problem and successfully obtained a set of rigorous elastic solution. By using this solution, he showed that surface shear force gives rise to fairly large maximum shearing stresses in pavements

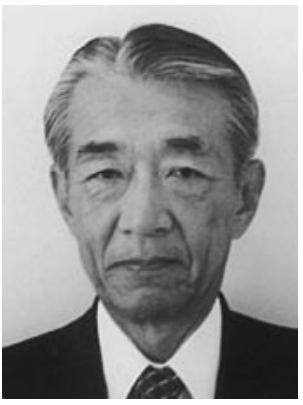
which are considered to be a major cause of the failure.

In 1965, he became a research associate at newly-established Civil Engineering Department at Tokyo Institute of Technology. Several years later, he changed his direction of research to studies on the bearing capacity of various soil layers. During the courses of his research relying on using small-scale models, he realized that there is a fatal shortcoming in using small-scale models for tackling the bearing capacity problems. It is not possible to produce the effect of self-weight of soil correctly in small-scale models. In order to overcome this problem, he thought of using a geotechnical centrifuge. Using the centrifuge, he carried out various studies including dynamic behavior of sand and also of silty soil. He was promoted to Professor at Tokyo Institute of Technology in 1981. He experienced two long stays at Cambridge University from 1978 to 1979 and from 1997 to 1998. In conducting various researches using centrifuge, he has developed close research collaboration with geotechnical engineering group at Cambridge University, which led to the award of CBE (Honorary Commander of the Most Excellent Order of the British Empire) in 2004. He has come up to many findings related to responses of soils subjected to various forms of loading, which are useful for design purposes. He was awarded the Japan Academy Prize in 2012. He served as President of Tokyo Institute of Technology from 1993 to 1997 and President of National Institution for Academic Degrees and University Evaluation from 1998 to 2009. 